\section{Massive Loss of Bile from the Nasobiliary Tube}

Therapeutic biliary endoscopy frequently involves temporary external bile drainage. Average bile production per day is $600 \mathrm{ml}$, but we report here a case in which it massively exceeded the normal range, leading to life-threatening symptoms.

A 52-year-old man with a known history of cirrhotic liver disease and chronic pancreatitis was admitted to our department, due to acute cholangitis. At endoscopic retrograde cholangiography (ERC), a single floating stone (diameter $10 \mathrm{~mm}$ ) was detected in the dilated common bile duct. A sphincterotomy was carried out and due to its small diameter, the stone was left for spontaneous evacuation, which was not confirmed before the patient was discharged. Three months later, he was readmitted with epigastric pain, jaundice, and fever. The total bilirubin level $3.6 \mathrm{mg} / \mathrm{dl}$, and enzymatic cholestasis (alkaline phosphatase, twice the normal level and $\gamma$-glutamyl transpepidase four times the normal level) were prominent laboratory findings. The ERC confirmed the presence of the stone. After it was removed using a Dormia basket, a nasobiliary drain was inserted, due to the presence of clinical signs of acute cholangitis and purulent bile. As a routine measure, the quantity of bile output was noted, and an uncommonly high production of bile, reaching $9250 \mathrm{ml} / 24 \mathrm{hr}$, was recorded. The patient rapidly developed hypovolemic shock, and a massive intravenous infusion of fluids to restore arterial pressure was required. The next day, the position of the nasobiliary drain was tested by fluoroscopy, again leading to a significant increase in bile secretion. The increase in bile secretion was temporary, and related to the intrabiliary or intravenous administration of iodinated contrast medium (Figure 1). Drugs known to induce choleresis were not given. The patient recovered quickly, and after normalization of the laboratory data, the nasobiliary drain was removed on the fourteenth day.

The reported case is an example of a puzzling coincidence of cholestasis and abundant choleresis. In humans, $40 \%$ of basal bile flow has been estimated to come from the bile duct epithelium (1). Proliferation of bile ducts and an increase in both the size and numbers of ductular epithelia is a well-known effect of semiobstructive choledochal stone disease, which is associated with a chronic increase in intraductal bile pressure (2). In addition, bile formation increases linearly with the increase in the number of ductular cells $(1,2)$, so that the secretory surface may have been substantially increased, and the effects of choleretic stimuli reinforced, in the present patient. It is also notable that the biliary hypersecretion occurred repeatedly after administration of iodinated contrast medium. Organic anions comprising iodine and secreted into bile, such as ioglycamide and iodipamide, have been shown to produce a choleretic effect, which was related to the osmotic potential of these agents $(3,4)$. The fact that contrastrelated choleresis lasted several days in this patient, and that it happened after the injection of contrast medium, secreted primarily by the kidneys, suggest a possible nonionic mechanism for choleresis. This observation indicates that, in patients with chronic bile flow impairment in whom an external bile drain is introduced, the use of contrast agents may be associated with a considerable loss of bile, so that its collection should be carefully observed.

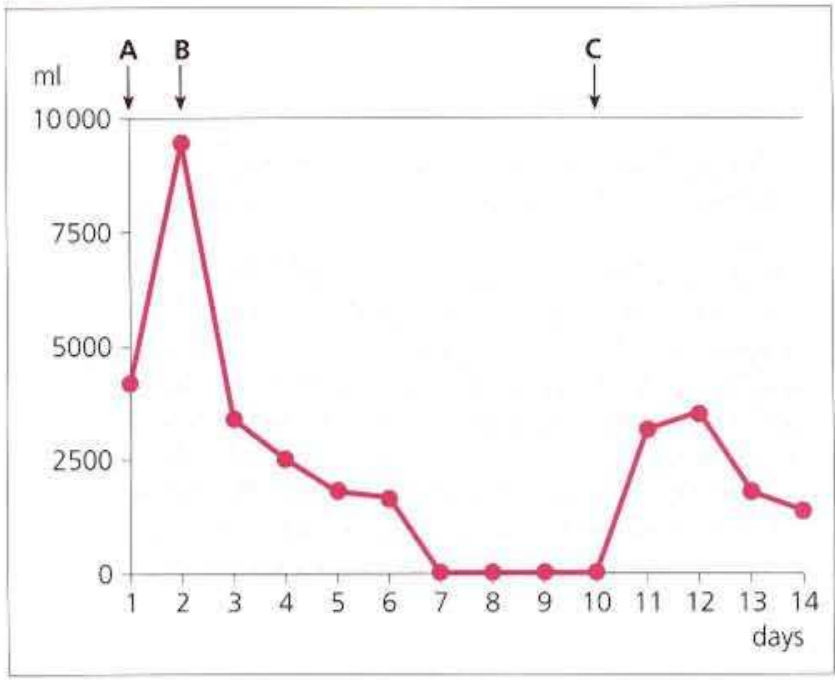

Figure 1: Volumes of daily bile collection through the nasobiliary drain Note the time relationship between contrast medium infusion and the volume of bile output. A: endoscopic retrograde cholangography; $B$ : contrast infused into the biliary tree during inspection of the nasobiliary drain position; C; contrast-enhanced computed tomography (the routes of contrast administration were intravenous and peroral). In all cases, the contrast medium was Uropolin (meglumine amidotrizoate, Polfa, Poland), $15-30 \mathrm{~g}$

M. Hartleb, E. Nowakowska-Dulawa, A. Kasicka-Jonderko,

A. Nowak

Dept, of Gastroenterology, Silesian Medical School, Katowice, Poland

\section{References}

1. Nathanson MH, Boyer JL. Mechanism and regulation of bile secretion. Hepatology 1991; 14: 551-66.

2. Slott PA, Liu MH, Tavoloni N. Origin, pattern, and mechanism of bile duct proliferation following biliary obstruction in the rat. Gastroenterology 1990; 99: 466-77.

3. Feld GK, Loeb PM, Berk RN, Wheeler HO. The choleretic effect of iodipamide. J. Clin Invest 1975; 55: 528-35.

4. Hoenig V, Preisig R. Organic-anionic choleresis in the dog: comparative effects of bromsulftalein, ioglycamide and taurocholate. Biomedicine 1973; 18: 23-30.

Corresponding Author

M. Hartleb, M. D.

Dept. of Gastroenterology

Silesian Medical School

ul. Medyków 14

40-752 Katowice

Poland

Fax: +48-3-1523119 